\title{
Predicting Recovery Potential (PREP)
}

\section{Prognose zur Erholung des Armes}

\author{
Tina Henneken
}

\begin{abstract}
Werde ich meinen Arm wieder wie zuvor gebrauchen können, werden Therapeuten häufig gefragt. Der Artikel beschreibt die Möglichkeiten und Grenzen eines wichtigen Prognosealgorithmus. Daneben zeigt er auch, wie sich die Prognoseübermittlung für die Therapie verwenden lässt.
\end{abstract}

\section{Was sind PREP und PREP2?}

PREP ist ein Akronym für Predicting REcovery Potential. Der PREP-Algorithmus gilt als der Goldstandard hinsichtlich der an Biomarkern orientierten Prognosen für die Erholung der oberen Extremität nach Schlaganfall. Hier werden Prädiktoren stufenweise abgearbeitet, nachdem zunächst die Charakteristika der Patienten erfasst wurden, die die Prognose am stärksten beeinflussen („Kraft“). Erst danach werden je nach der Ausprägung der Parese des Armes weitere Prädiktoren geprüft.

PREP wurde von Professor Cathy Stinear entwickelt und 2012 im Lancet Neurology publiziert [2]. Ursprünglich diente der PREP-Algorithmus der Stratifizierung von Patientengruppen für Studien, um die Ergebnisse aussagekräftiger belegen zu können und Responder und Non-Responder zu unterscheiden, wie von Horn und Lotze in diesem Heft beschrieben [1].

Der weiterentwickelte, neu modifizierte PREP2 wurde im November 2017 im Annals of Clinical and Translational Neurology veröffentlicht. Er erleichtert die Anwendung im klinischen Alltag erheblich, da die Verwendung von DTI-Bildern hier nicht mehr benötigt wird, auf der die Berechnung einer Fraktionalen Anisotropie (FA) für die PLIC (Posterior Limb of Internal Capsule) der kortikospinalen Bahnen (corticospinal tract $=$ CST) basierte. Dieser Teil wurde durch die Verwendung der NIHSS (National Institutes of Health Stroke Scale) ersetzt. Der Prädiktor „Alter“ wird einbezogen. Außerdem ist ein niedrigerer SAFE-Score von $\geq 5$ (Schulter-Abduktion, Finger-Extension = SAFE) ausreichend ( $\triangleright$ Abb. 1), um den Patienten als MEP + zu werten (Motorisch Evozierte Potenziale positiv). Das bedeutet, dass TMS (Transkranielle Magnetstimulation) bei weniger als einem Drittel der Patienten benötigt wird - anstatt der Hälfte wie bei PREP.
Genau wie beim PREP wird beim PREP2 in bis zu drei Schritten vorgegangen, um in den ersten 72 Stunden nach dem Schlaganfall eine Prognose des Outcomes nach zwölf Wochen errechnen zu können. Vier Outcome-Potenziale sind möglich: sehr gut, gut, limitiert, schwach [3].

\section{Durchführung PREP2}

\section{PREP2 in drei Schritten}

PREP2 ist geeignet für Patienten ab 18 Jahren, die einen akuten kortikalen oder subkortikalen Schlaganfall hatten, der mit einer Schwäche in Arm und Hand verbunden ist. Ausgenommen sind Patienten mit Schlaganfall im Kleinhirn, Aphasie oder kognitiver Beeinträchtigung.

Schritt 1 Innerhalb von 72 Stunden nach dem Schlaganfall wird der SAFE-Score durchgeführt. Dieser errechnet sich aus der Kraftmessung von Schulterabduktion plus Fingerextension ( $\triangleright \mathbf{A b b} \mathbf{2}$ ) und ergibt einen SAFE-Score von 0-10 Punkten, mit je 0-5 Punkten für Schulter und Finger.

Auswertung:

- Ist der SAFE-Score drei Tage nach dem Insult 5 oder höher, wird kein weiterer Test benötigt: Das Potenzial ist sehr gut ( $\mathbf{A b b} \mathbf{1} \mathbf{1})$.

- Ist der Patient über 80 Jahre alt und der SAFE-Score unter 8 , wird der funktionelle Outcome gut sein.

- Ist der SAFE-Score unter 5, geht man zu Schritt 2.

Schritt 2 Mittels Transkranieller Magnetstimulation testen wir die motorischen Bahnen zwischen betroffener Hirnhälfte und betroffenem Arm. Das dauert 15 Minuten und findet bis zu sieben Tage nach dem Schlaganfall statt ( Abb. 3). 
- Tab. 1 Einteilung des National Institutes of Health Scale (NIHHS)

\begin{tabular}{|c|c|}
\hline NIHSS-Score & Schweregrad des Schlaganfalls \\
\hline 0 & keine Schlaganfallsymptomatik \\
\hline $1-4$ & geringe Schlaganfallsymptomatik \\
\hline $5-15$ & moderate Schlaganfallsymptomatik \\
\hline $16-20$ & $\begin{array}{l}\text { moderate bis schwere Schlaganfall- } \\
\text { symptomatik }\end{array}$ \\
\hline $21-42$ & schwere Schlaganfallsymptomatik \\
\hline
\end{tabular}

Auswertung:

- Sind Motorisch Evozierte Potenziale abrufbar (MEP+), ist das Potenzial für den motorischen Outcome nach 12 Wochen gut.

- Sind keine Motorisch Evozierten Potenziale ableitbar (MEP-), wird Schritt 3 durchgeführt.

Schritt 3 Hier sieht man sich den NIHSS-Score an. Dieser Score wird immer bei Aufnahme auf einer Stroke Unit erhoben. Er gibt Aufschluss über den Schweregrad des Schlaganfalls. Dabei gilt: Je höher der Score, desto schwerer der Schlaganfall (> Tab. 1).

Auswertung:

- NIHSS < 7: limitiertes Potenzial für den funktionellen Outcome nach 12 Wochen

- NIHSS $\geq 7$ : schwaches Potenzial für den funktionellen Outcome nach 12 Wochen

\section{Therapiefokus entsprechend der Prognose}

PREP2-Prognosen können helfen, den Therapiefokus festzulegen:

- Sehr gutes Potenzial - Förderung normaler Gebrauch: Der Patient wird in zwölf Wochen eine (nahezu) vollständige Funktionserholung des betroffenen Armes erreichen. Im Fokus der Therapie stehen demnach ein forcierter Handeinsatz im Alltag sowie ein individuelles Eigenübungsprogramm (Heimübungsprogramm).

- Gutes Potenzial - Förderung der Funktion: Der Patient wird seinen Arm in zwölf Wochen bei fast allen Activities of Daily Living (ADLs) verwenden können. Die Therapie konzentriert sich auf Funktionsverbesserung, Kräftigung, Koordination, Feinmotorik, repetitives Training, Einsatz des Armes im Alltag unter Berücksichtigung der Sicherheit und Vermeidung von Kompensation.

- Limitiertes Potenzial - Förderung von Bewegung: Der Patient wird den Arm in zwölf Wochen bei einigen ADLs verwenden können. Die Wahrscheinlichkeit ist hoch, dass Modifikationen benötigt werden. Patient und Therapeut arbeiten am Erhalt und an der Verbes-

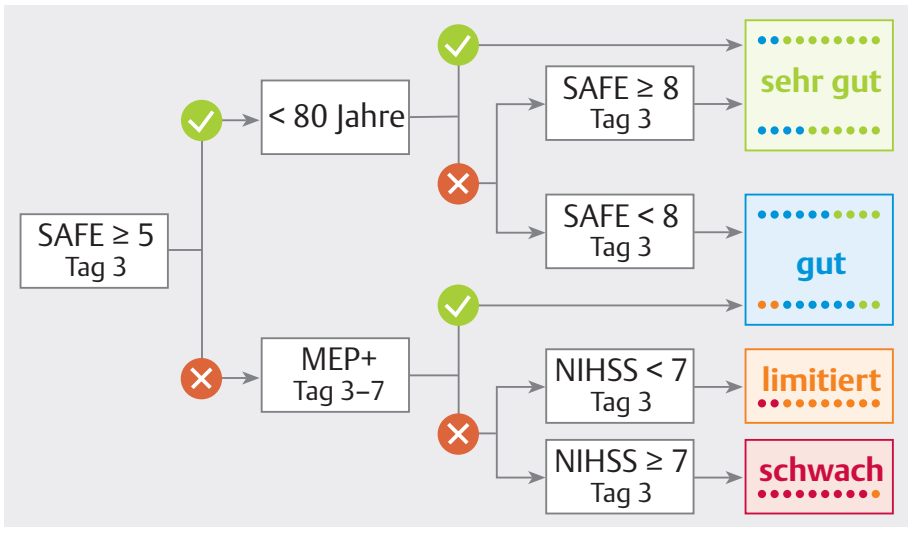

Abb. 1 Der PREP2-Algorithmus errechnet sich in drei Schritten und ergibt eine sehr gute, gute, limitierte oder schwache Prognose. (Quelle: T. Henneken; Umsetzung: Thieme Gruppe)
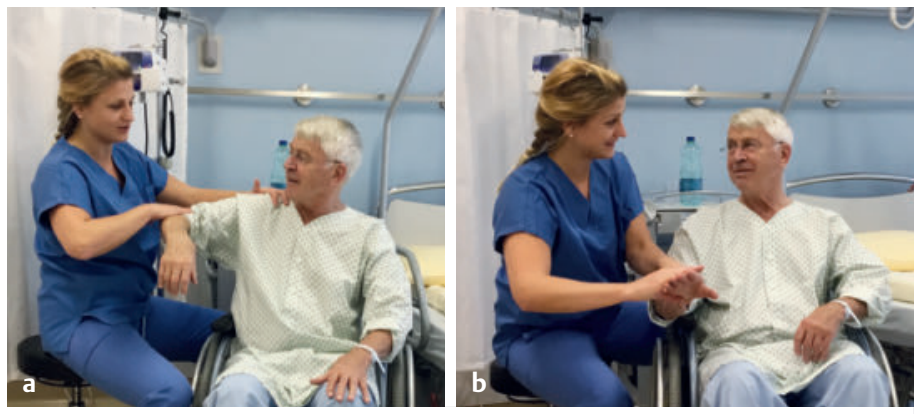

Abb.2 Zunächst erfolgt die Kraftmessung nach dem British Medical Council ( 0 = keinerlei palpierbare Muskelaktivität, komplette Lähmung; 5 = normale Kraft). Addiert man die Kraftmessung von Schulterabduktion mit der Fingerextension, erhält man einen SAFE-Score von 0 bis 10 Punkten. (Quelle: T. Henneken; Symbolbild)

serung von Kraft und Flexibilität, Assistenz bei Adaptionen und dem Gebrauch von Hilfsmitteln.

- Schwaches Potenzial - Förderung von Kompensation: Der Patient wird in zwölf Wochen sehr wenig Bewegungen im Arm zurückerlangen. Im Fokus der Therapie stehen damit die Prävention von Sekundärkomplikationen (Schmerz, Steifheit), Armhandling sowie Hilfe bei der Umsetzung von Einhändertechniken in den ADLs.

\section{Offen und sensibel kommunizieren}

Eine Prognose soll Hoffnung geben, aber keine falsche Hoffnung wecken. Eine schwache Prognose kann Betroffenen den Druck nehmen. Denn viele glauben, sie seien schuld daran, dass es nicht sehr viel besser wird. Eine sehr gute Prognose hingegen kann auch für manche eine schlechte Nachricht sein, weil sie eine hohe Erwartungshaltung haben. Es ist also wichtig zu wissen, dass wir in einem sehr sensiblen Moment vorsichtig den Rehabilitationsverlauf beeinflussen möchten. Für alle Patienten, die Schwierigkeiten haben, mit den Zukunftsaussichten umzugehen, sollte darüber hinaus selbstverständlich psychologische Unterstützung angeboten werden. Andere wie- 


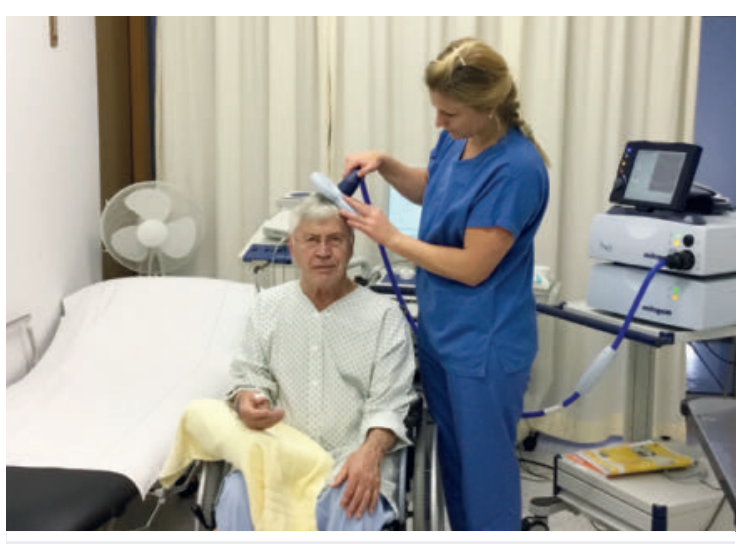

Abb. 3 Die Transkranielle Magnetstimulation (TMS) gehört zu den nichtinvasiven Untersuchungsmethoden. Sie löst Aktionspotenziale im Gehirn aus, indem sie das physikalische Prinzip der elektromagnetischen Induktion nutzt. (Quelle: T. Henneken; Symbolbild)

derum möchten keine Prognose erhalten. Das muss offen kommuniziert werden und gilt es zu respektieren.

Die Prognose mitteilen Klienten und deren Angehörigen eine Prognose mitzuteilen, ist mehr als nur die Benachrichtigung über das Ergebnis. Zudem sind Prognosen keine absolute Garantie, denn manche Menschen entwickeln sich besser und manche schlechter als erwartet. Aus diesem Grund ist es wichtig, Begriffe wie „nahezu normal“, „sehr wahrscheinlich“, „wahrscheinlich“ oder „unwahrscheinlich“ und „sehr unwahrscheinlich“ zu verwenden. Vermeiden sollte man Phrasen, die dem Klienten das Gefühl vermitteln, dass nichts besser werden wird wie „schlecht (bleiben)“, „nicht gut“, „nicht normal“, „, nie wieder“ oder „keine Chance“. Eine sehr gute oder gute Prognose mitzuteilen, ist nicht schwierig. Aber die Betroffenen, die ein limitiertes oder schwaches Potenzial haben, benötigen eine bedachte Aufklärung und haben oft viele Fragen. In diesen Fällen hilft folgendes Schema für die Mitteilung:

- 1. Ergebnis: Mitteilung Prognose. Das Potenzial ist ...

- 2. Erwartung: Bewegung wird ... sein. Fokus der Therapie ist ...

- 3. Aufgabe: Auftrag. Übungen neben Therapie sind ...

- 4. Erklärung: Momentaner Status. Keine Garantie ...

\section{Wie genau ist PREP2?}

PREP2 wurde in einer Studie mit 207 von Schlaganfall betroffenen Patienten erhoben, die in den ersten drei Tagen nach dem Schlaganfall eingeschlossen wurden. Insgesamt war die Prognose für den Outcome der oberen Extremität für 156 von 207 Patienten korrekt (75\%). Von den übrigen 51 Patienten war die PREP2-Prognose für 35 Patienten zu optimistisch und für 16 zu pessimistisch. Den 35 zu optimistisch eingeschätzten Patienten wurde ein sehr guter Outcome errechnet, aber sie erreichten stattdessen einen guten ( $n=25)$ oder sogar nur limitierten $(n=1)$ Outcome. Alle Patienten, die zu pessimistisch eingeschätzt wurden, hatten ein gutes Potenzial, erreichten aber einen sehr guten Outcome $(n=14)$. Durch den Zusammenschluss von der Berechnung des SAFE-Scores eines Patienten mit dessen Alter erreichte man eine verbesserte Genauigkeit der Prognose von $68 \%$ auf $78 \%$. Dies betrifft Patienten mit einem sehr guten oder guten Potenzial für den Outcome der oberen Extremität zwölf Wochen nach dem akuten Schlaganfall. Für Patienten mit einem SAFE-Score $\leq 5$ fand man heraus, dass allein der NIHSS ohne MEP-Status den Outcome nur zu 55 \% Genauigkeit für den guten oder schwachen Outcome prognostiziert. Durch die TMS-Biomarkerinformation verbessert sich die Prognosegenauigkeit für diese Patienten auf $70 \%$. Dies zeigt die Wichtigkeit der Testung der kortikospinalen Bahnen auf, gerade für die schwerer betroffenen Patienten [3].

\section{Klinischer Alltag PREP2}

Der klinische Alltag sieht natürlich häufig ganz anders aus als eine Versuchssituation. Hier sind Effizienz und Einfachheit der Vorgehensweise für den Anfang einer Implementierung von PREP2 unumgänglich. Zwar ist die Messung der MEPs weder schwierig noch zeitaufwendig, jedoch muss auf der einen Seite das Gerät vorhanden sein und auf der anderen Seite die Benutzung unter einem genauen Protokoll stattfinden (Patienten- und Angehörigenaufklärung, SAFE-Score < 5, Sicherheitscheckliste TMS, MEPMessung innerhalb von 3-7 Tagen).

Viele haben Hemmungen, eine „ungünstige“ Prognose mitzuteilen. Aus der Erfahrung im klinischen Alltag ist das aber nicht nötig, da gerade die schwer betroffenen Patienten, die zunächst wirken, als hätten sie ein wenig erfolgversprechendes Potenzial, mit PREP2 durch die Messung der MEPs und Nutzung der NIHSS in eine „bessere“ Gruppe aufsteigen. Die Patienten, die zu solchen „Mischgruppen“ gehören, sind die, die wir ohne PREP2 entweder unterschätzen oder überfordern könnten. Es können z. B. Patienten sein, die ohne TMS mit einem SAFE-Score $<5$ zunächst nach einem schwachen Potenzial aussehen, aber bei MEP + sogar eine gute Prognose und auch bei MEP- mit NIHSS $\geq 7$ dennoch eine limitierte Prognose erhalten würden. Sowohl die Patienten und die Angehörigen als auch das involvierte Klinikpersonal (Therapeuten, Pflegende, Ärzte) empfinden ein solches Ergebnis als besonders positiv überraschend. Ein Hinarbeiten auf ein nachweislich realistisches Ziel bringt sehr viel mehr Freude an den Fortschritten und dem Eigentraining als ein Hinarbeiten auf ein ungewisses, unrealistisches oder unterschätztes Ziel. 


\section{PREP2 im Alltag aus Sicht einer Therapeutin}

\section{Beispiel 1}

Der Patient wird an einem Dienstagnachmittag auf der Stroke Unit aufgenommen (Tag 1). Er ist 88 Jahre alt. Die Diagnose bestätigt den Verdacht eines Schlaganfalls: ein Capsula-interna-Infarkt links mit distal betonter Hemiparese rechts (NIHSS 6). Am Mittwoch (Tag 2) wird die Therapie zugewiesen. Trotz leichter Erschöpfung des Patienten kann der SAFE-Score erhoben werden: SAFE-Score = 3. Der Patient weist eine ausgeprägte distale Parese auf, Schulterabduktion $3 / 5$, Fingerextension 0/5.

Die Therapie verläuft wie gewohnt. Der Patient lernt das Armhandling, erste Übungen können schon im Sitz auf der Bettkante, später im Rollstuhl gemacht werden. Am Freitag (Tag 4) ist der Patient stabil und wird auf die Normalstation verlegt. Zunächst wird der Patient gefragt, ob er eine Prognose für den Outcome seines Arms nach zwölf Wochen erhalten möchte, denn zuvor stellte er bereits die Frage: „Wird der Arm wieder werden?“ Es wird ihm erklärt, dass dies durch eine nichtinvasive Messung der absteigenden Bahnen vom Gehirn zur betroffenen Hand möglich ist. Er wird ein Klopfen durch die Magnetspule spüren, wenn diese sich entlädt, was wir aber ankündigen und vorführen werden. Diese Untersuchung dauert circa 15 Minuten. Er wünscht eine Prognose zu erhalten, und die TMSCheckliste und -Einwilligung werden durchgearbeitet und unterschrieben.

Die Messung der MEPs wird am Montag durchgeführt (Tag 6): MEP- ( $\mathbf{A}$ bb. 4). Nun muss der NIHSS vom Tag der Aufnahme herangezogen werden, NIHSS $=6$. Der Wert ist $<7$ und bedeutet, dass das Potenzial nicht schwach, sondern limitiert ist. Nur TMS oder nur NIHSS reichen nicht aus, denn wenn der Patient MEP + gehabt hätte, wäre sein Potenzial sogar gut gewesen. Mithilfe des Vier-Punkte-Schemas kann ihm die Prognose genau erklärt werden.

Vier-Punkte-Schema:

- 1. Ergebnis: Ihr Arm und Ihre Hand werden in den nächsten drei Monaten höchstwahrscheinlich limitiert Besserungen zeigen.

- 2. Erwartung: Sie können erwarten, dass Sie ein wenig Bewegung zurückerlangen werden. Das heißt, die Feinmotorik wird sicherlich eine große Herausforderung bleiben. Der Fokus der Reha wird sein, die Kraft und Flexibilität des Armes und der Hand zu verbessern und Sie mit Adaptionen und Tricks auszustatten, damit Sie den Arm bestmöglich werden verwenden können.

- 3. Aufgabe: Sie werden auch selbst außerhalb der Therapie viel üben müssen, um das Ergebnis zu verbessern. Wir zeigen Ihnen Wege, wie Sie selbst den Arm im Alltag gut einbinden können.
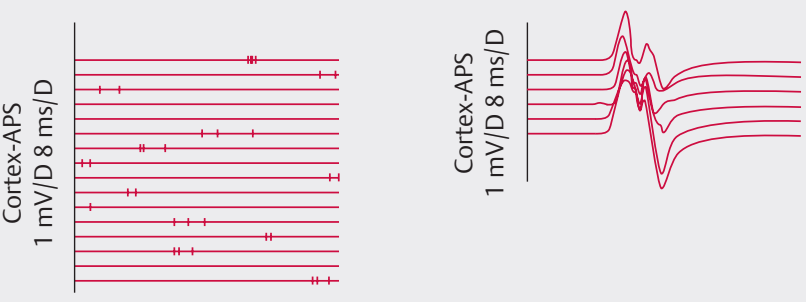

Abb. 4 TMS-Diagnostik von Patient 1. Es sind keine MEPs vom Kortex links (M1) über die absteigenden kortikospinalen Bahnen ableitbar.(Quelle: T. Henneken; Umsetzung: Thieme Gruppe)

- 4. Erklärung: Diese Prognose basiert auf Ihrem aktuellen Status. Es ist keine Garantie, da sich einige Menschen mehr und andere weniger gut erholen.

Der Patient möchte, dass seine Gattin dabei ist. In Neuseeland wird als Teil von PREP2 zur Unterstützung der Mitteilung einer Prognose ein Tablet mit den MRT-Bildern verwendet ( $\triangleright$ Abb.5), das geht aber aus Datenschutzgründen in Österreich nicht. Es wäre hilfreich gewesen, denn der Patient ist zwar zufrieden mit dem Ergebnis und vielleicht sogar ein bisschen erleichtert, aber seine Frau kann das Ergebnis noch nicht ganz begreifen. Es können vielleicht für den Moment nicht alle Fragen geklärt werden, aber in den darauffolgenden Tagen versteht die Frau des Patienten das Ergebnis und kann motivierend am Reha-Prozess teilnehmen. Das Ziel des Patienten war es, den betroffenen Arm in Zukunft als Hilfshand, z. B. beim Öffnen einer Flasche, eines Duschgels oder zum Gegenhalten beim Schneiden von Gemüse, verwenden zu können. Das Ziel hat er erreicht. Selbstverständlich hat er sich zwölf Wochen nach dem Schlaganfall weiter verbessert.

\section{Beispiel 2}

Die Patientin, 73 Jahre, hat eine seit dem Vortag zunehmende Schwäche der linken Seite (Tag 2). In der Bildgebung zeigt sich ein Marklagerinfarkt rechts. Am selben Tag kann der SAFE-Score erhoben werden: SAFE-Score $=4$ (Tag 2). Ihre Schulterabduktion ist $2 / 3$ und die Fingerextension 2/3. Zwei Tage darauf wird die Patientin in stabilem Zustand auf die Normalstation verlegt (Tag 4). Mit einem SAFE-Score von < 5 ist der nächste Schritt die TMS-Diagnostik zur Darstellung der MEPs, wenn die Patientin dies wünscht. Wir erklären ihr, dass wir im jetzigen Zeitfenster bis sieben Tage nach dem Schlaganfall eine Prognose für ihren Outcome der oberen Extremität stellen können. Damit können wir vorhersagen, was sie mit hoher Wahrscheinlichkeit erreichen können wird. Um die genaue Berechnung des Algorithmus vornehmen zu können, müssen wir eine Untersuchung der absteigenden Bahnen mit TMS bei ihr vornehmen. Die Patientin versteht die Instruktionen und willigt ein (Tag 4). Die TMS-Diagnostik ergibt $\mathrm{MEP}+(\triangleright$ Abb. 6). Die Patientin hat eine gute Prognose. 

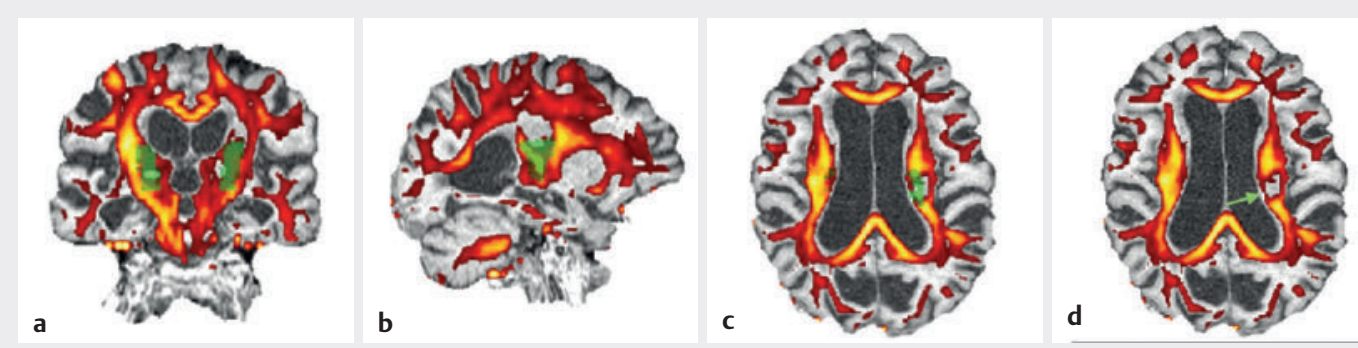

Abb. 5 Beispiel von Patient 1, diffusionsgewichtete Bildgebung. Darstellung der PLIC (Posterior Limb Internal Capsule) in grün in Ebenen frontal (a), sagittal (b) und transversal (c). Grüner Pfeil (d) zeigt Infarktgebiet im Bereich der Capsula interna. (Quelle: T. Henneken)
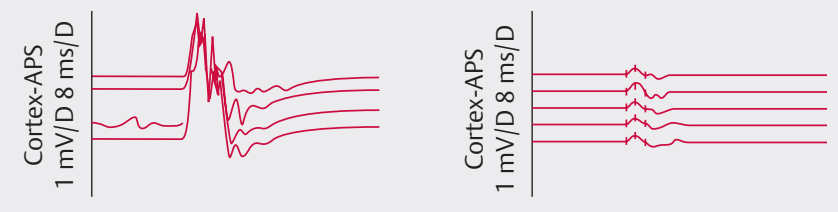

Abb. 6 TMS-Diagnostik von Patientin 2. Es sind MEPs vom Kortex rechts (M1) über die absteigenden kortikospinalen Bahnen ableitbar. (Quelle: T. Henneken; Umsetzung: Thieme Gruppe)

Vier-Punkte-Schema:

- 1. Ergebnis: Ihr Arm und Ihre Hand werden in den nächsten drei Monaten höchstwahrscheinlich gute Besserungen zeigen.

- 2. Erwartung: Sie können erwarten, dass Sie in zwölf Wochen Ihren Arm und Ihre Hand bei nahezu allen Tätigkeiten im Alltag einsetzen lernen werden. Der Fokus der Reha wird sein, den Arm und die Hand zu kräftigen, die Koordination zu trainieren und an der Feinmotorik zu arbeiten. Sie werden auch Bewegungen repetitiv üben.

- 3. Aufgabe: Sie werden außerhalb der Therapien viel üben müssen, um das Ergebnis zu verbessern. Es wird anstrengend werden, den betroffenen Arm forciert und unentwegt im Alltag einzusetzen, ohne den anderen dabei zu Hilfe zu nehmen. Achten Sie darauf, es von heute an zu üben.

- 4. Erklärung: Die Prognose basiert auf Ihrem aktuellen Status und ist keine Garantie, da sich Menschen unterschiedlich gut erholen.

Für die Patientin ist es eine sehr gute Nachricht, denn im Moment kann sie ihre Hand noch nicht adäquat verwenden. In der Therapie wird ein repetitives Training gestartet. Mit zusätzlichen Eigenübungen für Kraft (Stütz) und Bewegungsausmaß (Schulter) kann sie die Hand bereits eine Woche später im Alltag verwenden, anfangs noch mit Griffverdickungen und nicht ausdauernd. Für die Patientin steht fest, dass die Prognose sie erst richtig motiviert hat.
Eigentlich hat es sogar ihren Ehrgeiz geweckt, das Ergebnis zu übertreffen, sagt sie. Aber sie weiß, dass sie auch danach noch weiter Fortschritte machen wird.

\section{Resümee}

Beide Patienten sind typische Beispiele für Betroffene, die sich selbst ohne die Prognose hinsichtlich des motorischen Outcomes schlechter eingeschätzt hätten. Auch das Klinikpersonal reagiert in solchen Fällen positiv überrascht, was zusätzlich motivierend auf die Patienten wirken kann. Und auch wenn für den ersten Patienten der Outcome nicht so gut klingt wie für die zweite Patientin, so nimmt es ihm augenblicklich den Druck, mehr erreichen zu müssen, als er für möglich halten kann. Mit Prognosen lernen wir, mehr noch das Mögliche und nicht das Unmögliche zu verlangen und uns unserer Sache sicher zu fühlen.

Eignung für die Prognose Nicht sehr viele Patienten eignen sich für eine Prognose, da sie nicht die Voraussetzungen erfüllen. Ein großer Teil erhält Interventionen wie Thrombolysen und/oder Thrombektomien. Ein weiterer Teil von Patienten nach Schlaganfall ist bereits vor dem Schlaganfall multimorbid. Vor allem die schwerer betroffenen Patienten mit einem SAFE-Score $<5$, für die eine Prognose eine durchaus wichtige Rolle spielen kann, stellen nur eine geringe Anzahl dar. Die Patienten mit einer guten oder sehr guten Prognose sind wohl die größte Gruppe, die mit einer gewissen Erfahrung und Expertise von Therapeuten wahrscheinlich vollkommen richtig eingeschätzt werden würden.

Zweifel an der Prognose Prognosen werden häufig angezweifelt. Das ist auch gut so. Nichts soll unsere gesunde Skepsis verdrängen. Noch ist die Genauigkeit nicht hoch genug, sodass es wie bei der Wetterprognose nur eine Wahrscheinlichkeit bleibt. Mit PREP2 können wir Patienten in Prognosegruppen einteilen und Therapieentscheidungen vereinfachen. Wir können zum richtigen Zeitpunkt mit den geeigneten Mitteln die Rehabilitation des Patienten effizient unterstützen. 
Individuelle Therapievarianz Die Frage lautet nach wie vor oft, ob eine wenig erfolgversprechende Prognose einem Menschen nicht die Hoffnung nähme. Und das ist wohl auch neben der Skepsis gegenüber der Genauigkeit einer Prognose eine sehr große Hürde für Therapeuten, Prognosen zu verwenden. Ein Patient sagte einmal darauf, dass einem die Hoffnung genommen werden würde, wenn einem etwas vorgemacht wird. Die Arbeit mit Menschen bleibt wie immer individuell zu betrachten. Daher ist der PREP2-Algorithmus trotz der Verwendung eines kostspieligen TMS-Gerätes eine gute Möglichkeit, Prognosen im Alltag einzusetzen, da Patienten in vier Prognosegruppen eingeteilt werden und keine expliziten Funktionsoutcomes vorhergesagt werden. Das ermöglicht eine individuelle Varianz für die Therapie.

Genauigkeit Es werden größere und multizentrische Studien benötigt, um die Genauigkeit zu verbessern. Untersuchungen zu verschiedenen Prädiktoren in diversen Kombinationen können die Entwicklung vorantreiben.

\section{Autorinnen/Autoren}

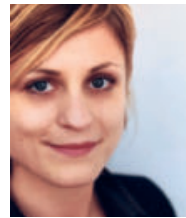

\section{Tina Henneken, MSc}

Arbeitet seit 2005 in der Neurorehabilitation in Deutschland, Österreich und der Schweiz. Das Jahr 2013 verbrachte sie in Australien und Neuseeland, wo sie u. a. mit Assoc. Prof. Dr. Cathy Stinear arbeitete und wo ihr Interesse für Schlaganfallforschung geweckt wurde, im Speziellen für das Prognostizieren des Funktionsoutcomes der oberen Extremität nach akutem Schlaganfall. Von 2014-2017 absolvierte sie ein Masterstudium der Neurowissenschaften und Neurorehabilitationsforschung. Ihr Masterthesen-Projekt widmete sie der Validierung eines simplen „Bedside“-Screenings zur Prognosestellung für den Funktionsoutcome nach Schlaganfall (Rule of Thumb). Eines ihrer Hauptinteressen gilt der Implementierung von Forschungsergebnissen in den therapeutischen Alltag.
Korrespondenzadresse

\section{Tina Henneken, MSc}

Ergotherapeutin, Abteilung für Neurologie

Universitätsklinikum Tulln

Karl Landsteiner Universität für Gesundheitswissenschaften

Alter Ziegelweg 10

3430 Tulln

Österreich

E-Mail: christina.henneken@tulln.lknoe.at

http://www.tulln.lknoe.at

Literatur

[1] Horn U, Lotze L. Prognosestellungen in der Neurorehabilitation: Möglichkeiten und Grenzen. neuroreha 2019; 11(2): 53-58

[2] Stinear CM, Barber PA, Petoe $M$ et al. The PREP algorithm predicts potential for upper limb recovery after stroke. Brain 2012; 135(Pt 8): 2527-2535

[3] Stinear CM, Byblow WD, Ackerley S] et al. PREP2: A biomarker-based algorithm for predicting upper limb function after stroke. Ann Clin Transl Neurol 2017; 4(11): 811-820. doi: $10.1002 / \operatorname{acn} 3.488$

Bibliografie

DOI https://doi.org/10.1055/a-0868-0554 neuroreha 2019; 11: 72-77

(c) Georg Thieme Verlag KG Stuttgart · New York ISSN 1611-6496 